INRA Prod. Anim., 1992, $5(1), 37$ - 42
A. HODEN, L. DELABY, B. MARQUIS

INRA Station de Recherches sur la Vache laitière 35590 Saint-Gilles

\title{
Pois protéagineux comme concentré unique pour vaches
laitières unique pour vaches
laitières
}

\author{
Les légumineuses à grosses graines ou protéagineux sont des aliments \\ riches en énergie contenant au moins $20 \%$ de matières azotées. Malgré le \\ développement récent des surfaces cultivées, leur utilisation par les \\ ruminants a été jusqu'ici limitée en raison notamment de l'utilisation bien \\ maîtrisée du tourteau de soja importé. Les conditions d'utilisation et la \\ valorisation de ces aliments par les ruminants sont aussi peu connues. Ces \\ sources azotées métropolitaines peuvent-elles convenir aux vaches \\ laitières? Et dans quelles conditions?
}

Les protéagineux (pois, lupin, féverole) et principalement le pois représentent une source non négligeable de protéines métropolitaines puisqu'il en est produit actuellement plus de 3 millions de tonnes par an (source UNIP-ITCF 1990). Ce déve-

\section{Résumé}

Deux rations complètes équilibrées à base d'ensilage de maïs comportant des apports iso-énergétiques d'aliments concentrés témoin classique (1 UFL/kg MS et 115 PDIE/UFL) ou expérimental (pois protéagineux) offertes en quantités limitées ont été comparées. Les apports nutritifs (UFL - PDI) ont été prédéterminés de façon à satisfaire sans excès les besoins des animaux. Selon un schéma expérimental en inversion comportant 2 périodes de 7 semaines, un total de 21 vaches en milieu de lactation ( $80 \%$ primipares) réparties en 2 lots a reçu l'une ou l'autre de ces rations.

Pour des apports moyens de 5,4 kg MS de concentré témoin ou de $4,6 \mathrm{~kg}$ MS de pois protéagineux, les quantités ingérées totales ont été identiques $(3,08 \mathrm{~kg} \mathrm{MS} \% \mathrm{~kg}$ de poids vif). Les apports énergétiques ont été comparables $(15,6 \mathrm{UFL} / \mathrm{j})$ et ceux de PDI ont été sensiblement plus faibles pour la ration comportant le pois protéagineux. Les bilans énergétiques ont été légèrement excédentaires, surtout pour le traitement expérimental (+ 0,7 UFL). Les bilans PDI sont à l'opposé déficitaires, en particulier pour la ration comportant le pois protéagineux ( $-130 \mathrm{~g}$ contre $-55 \mathrm{~g}$ pour le témoin).

Pour les 2 traitements, les productions de lait, de matières grasses et de protéines ont été comparables et en moyenne de $23,5 \mathrm{~kg}, 948 \mathrm{~g}$ et $692 \mathrm{~g}$ par jour respectivement. La composition du lait a aussi été identique mais les taux protéiques ont été faibles par rapport aux taux butyreux (30 vs $41 \mathrm{~g} / \mathrm{kg}$ respectivement). Les vaches les plus fortes productrices ont cependant diminué plus fortement leur production de lait $4 \%$ $(\mathrm{P}<0,07)$, de matières grasses $(\mathrm{P}<0,02)$ et le taux butyreux $(\mathrm{P}<0,02)$ lorsqu'elles ont reçu le pois protéagineux.

En conclusion, il est possible de valoriser des quantités importantes de pois protéagineux jusqu'à des niveaux de production de 25 à $30 \mathrm{~kg}$ de lait par jour. Pour les productions supérieures, il sera nécessaire d'apporter simultanément des protéines de qualité. loppement récent (doublement de la collecte au cours des 4 dernières années) est à mettre essentiellement à l'acquis du soutien communautaire octroyé aux utilisateurs.

Ces protéagineux présentent l'avantage d'être riches en énergie $(1,16$ à $1,25 \mathrm{UFL} / \mathrm{kg} \mathrm{MS})$ et de contenir de 25 à $40 \%$ de matières azotées ( $\mathrm{N} \mathrm{X}$ 6,25 de la MS) (INRA 1988). Leurs conditions d'utilisation par les ruminants et en particulier par les vaches laitières ont été peu diffusées jusqu'à ces dernières années. Ceci tient essentiellement à la simplicité et au succès de l'emploi des sources de protéines importées à des prix compétitifs (tourteau de soja en particulier). Divers travaux ont cependant été réalisés par l'INRA (Tisserand 1977 ; Hoden 1982 ; Huguet et al 1983 ; Emile et al 1991) et par les Organismes de Développement (Duchateau et Dedenon 1989 ; Chenais et Espinasse 1989 ; Weiss et Leboucher 1989 ; Rivière et Soulard 1990) sur l'utilisation des protéagineux. Concernant le pois, les essais ont généralement été réalisés en l'apportant comme concentré de type "production", la ration de base (ensilage de maïs) ayant été au préalable "corrigée" à partir de tourteaux (soja tanné ou non). Par ailleurs, dans la plupart des cas, les expérimentations réalisées chez des vaches en milieu de lactation se sont traduites par des niveaux d'alimentation excédentaires par rapport aux besoins des animaux. Il n'est alors pas aisé de juger de la valorisation réelle des aliments complémentaires, à partir d'un seul niveau d'apports nutritifs, compte tenu des phénomènes de rende- 
ments marginaux aux alentours de la couverture stricte des besoins (INRA 1988).

Afin de compléter les références acquises, il nous est apparu nécessaire d'effectuer une comparaison dans laquelle le pois protéagineux serait apporté en quantité relativement importante et à un niveau correspondant au mieux à la couverture des besoins des animaux. Par rapport à une complémentation témoin classique iso-énergétique satisfaisant les besoins PDI, l'objectif était de mettre en évidence les éventuelles limites pratiques d'utilisation du pois protéagineux comme source de protéines. Pratiquement, la comparaison a été réalisée avec une "ration complète" à base d'ensilage de maïs complémentée uniquement à partir de pois protéagineux apportés sur la base de la couverture des besoins énergétiques des animaux sans préjuger de sa valeur azotée. Il n'a malheureusement pas été possible de comparer plusieurs niveaux d'apports nutritifs aux alentours des recommandations. Cet essai s'est déroulé à l'INRA de Rennes durant l'hiver 19891990 avec des vaches en milieu de lactation.

\section{Conditions expérimentales}

Selon un schéma expérimental en inversion comportant 2 périodes de 7 semaines chacune, 2 lots de 11 et 10 vaches laitières $(80 \%$ de primipares) ont été utilisés pour comparer une complémentation unique à partir de pois protéagineux (traitement expérimental) par rapport à celle réalisée de façon classique (traitement témoin). De l'ensilage de maïs d'excellente qualité $(40,3 \% \mathrm{MS}$, $43,6 \%$ grains) et $1 \mathrm{~kg}$ de foin ont été apportés à volonté selon la technique de la "ration complète" jusqu'à un niveau maximum de $12 \mathrm{~kg}$ MS par vache et par jour, l'apport énergétique permettant d'assurer la production de $14 \mathrm{~kg}$ de lait. La limitation volontaire des quantités offertes de la ration de base avait pour objectif de respecter au mieux la couverture stricte des besoins des animaux (INRA 1988) compte tenu du schéma de complémentation appliqué. Les quantités d'aliments concentrés introduites dans l'ensilage de maïs ont été constantes intra-périodes et prédéterminées en supposant une décroissance de la production laitière de 1,5\% par semaine (Faverdin et al 1987).

Pour le traitement expérimental, le pois protéagineux broyé grossièrement a été apporté comme seul aliment complémentaire. Il assure ainsi simultanément les rôles de concentré "correcteur" de la ration de base et de concentré "production" en fonction de la quantité de lait individuelle. Dans ces conditions, il a été introduit $1 \mathrm{~kg}$ de pois protéagineux à raison de $2 \mathrm{~kg}$ de lait entre la quantité de lait permise par les UFL de la ration de base $(14 \mathrm{~kg})$ et le niveau de production moyen attendu pour chacune des périodes. Pratiquement, ceci a correspondu à mélanger 6 et $5 \mathrm{~kg}$ de pois protéagineux à l'ensilage de maïs lors des périodes 1 et 2 respectivement.

Pour le traitement témoin, les quantités d'aliment complémentaire ont été apportées de façon iso-énergétique par rapport à l'expérimental. Un mélange de diverses sources énergétiques (pulpes de betteraves, céréales et co-produits) et protéiques (tourteau de soja, luzerne déshydratée) a été constitué afin d'assurer aussi la couverture stricte des besoins PDI des animaux. Ceci a correspondu à un mélange d'environ $1 \mathrm{UFL} / \mathrm{kg} \mathrm{MS}$ (115 g PDI/UFL), introduit à raison de 7 et $6 \mathrm{~kg}$ respectivement aux périodes 1 et 2 . L'apport de tourteau pur a alors été d'environ 1 et $0,8 \mathrm{~kg}$.

Les principales conditions expérimentales concernant la valeur nutritive (INRA 1988) et la composition des aliments ainsi que la constitution des rations complètes sont rapportées au tableau 1. Ces rations permettaient également d'assurer les besoins en azote fermentescible (apport d'urée) ainsi que ceux en minéraux et vitamines.

Les critères d'appariement des animaux ont été le rang de lactation ( 2 multipares par lot), le stade de lactation ( $42 \pm 10$ jours), la production laitière $(28,9 \pm 5,0 \mathrm{~kg})$, la composition du lait $(40,7 \pm 5,6 \mathrm{~g} / \mathrm{kg}$ de taux butyreux et $28,1 \pm 1,9 \mathrm{~g} / \mathrm{kg}$ de taux protéique) et le poids vif $(565 \pm 47 \mathrm{~kg})$ observés au cours d'une pré-expérience de 3 semaines.

Les mesures individuelles des quantités de lait produites ont été effectuées chaque jour, et la composition du lait (taux butyreux et de protéines) a été déterminée 3 jours par semaine ( 6 traites). Les animaux ont été pesés uniquement en pré-expérience et au cours des 2 dernières semaines de chaque période à jours et heures fixes. Les quantités ingérées ont été contrôlées quotidiennement au niveau de chacun des lots de 10 et 11 vaches qui étaient en stabulation libre sur aire paillée.

L'ensemble des données individuelles a été traité par analyse de variance en utilisant la procédure GLM de SAS (SAS 1987) qui permet d'obtenir l'estimation de la moyenne des traitements en prenant en compte les effets des périodes et des individus.

\section{Résultats - discussion}

\section{*Quantités ingérées et apports nutritifs}

Les niveaux d'ingestion (tableau 2) des 2 rations complètes sont comparables $(3,1 \mathrm{~kg} \mathrm{MS} / 100 \mathrm{~kg}$ de poids vif). Ils correspondent aux capacités d'ingestion habituellement observées (Hoden et al 1988) compte tenu des caractéristiques des animaux (âge, production, poids vif). Ils auraient été plus élevés si l'ensilage de maïs n'avait pas dû être limité à partir de la 2ème période afin d'éviter une suralimentation excessive. Cette limitation n'a pas été nécessaire en 1ère période car les vaches n'avaient pas encore atteint leur capacité d'ingestion maximum en début d'essai (42ème jour de lactation en moyenne). Dans ces conditions, les quantités ingérées ont été plafonnées aux environs de $17,5 \mathrm{~kg}$ MS totale durant l'ensemble de la 2ème période. Les proportions d'aliments complémentaires (urée et minéraux compris) dans la ration totale ont été en moyenne de 33 et $29 \%$ pour les traitements témoin et expérimental respectivement. Pour le traitement témoin, les quantités de 
Tableau 1. Utilisation des pois protéagineux par les vaches laitières

Composition chimique et valeur nutritive des aliments ")

\begin{tabular}{|c|c|c|c|c|c|c|c|}
\hline \multirow{2}{*}{ Aliments } & \multicolumn{7}{|c|}{ Par kg de MS } \\
\hline & UFL & $\begin{array}{c}\text { PDIN } \\
(\mathrm{g})\end{array}$ & $\begin{array}{c}\text { PDIE } \\
(\mathrm{g})\end{array}$ & $\begin{array}{c}\text { MAT } \\
(\mathrm{g})\end{array}$ & $\begin{array}{c}\text { Cellulose } \\
\text { brute } \\
\text { (g) }\end{array}$ & $\begin{array}{l}P \\
\text { (g) }\end{array}$ & $\begin{array}{l}\mathrm{Ca} \\
(\mathrm{g})\end{array}$ \\
\hline Ensilage de maïs & 0,91 & 52 & 72 & 85 & 219 & 2,0 & 3,0 \\
\hline Foin de graminées & 0,73 & 104 & 90 & 168 & 267 & 2,5 & 4,0 \\
\hline Concentré Témoin ${ }^{(2)}$ & 1,02 & 121 & 113 & 182 & 118 & 6,4 & 7,9 \\
\hline Pois protéagineux & 1,16 & 151 & 103 & 250 & 59 & 4,6 & 0,9 \\
\hline Urée & - & 1472 & - & 2875 & - & - & \\
\hline
\end{tabular}

1) Selon Tables INRA 1988, et dosage des matières azotées $(\mathrm{N}$ x 6,25) et cellulose brute.

(2) Composition $(\%)$ : pulpes de betteraves $(26)+$ orge $(21,5)+$ son fin de blé $(21,5)+$ luzerne déshydratée $(8,5)+$ tourteau de soja $48(14)+$ mélasse $(4,5)+$ minéraux $(4)$.

\section{Composition des rations complètes mélangées ${ }^{(1)}$}

\begin{tabular}{|c|c|c|c|c|}
\hline \multirow[t]{2}{*}{ Traitement } & \multicolumn{2}{|c|}{ Témoin } & \multicolumn{2}{|c|}{ Expérimental } \\
\hline & I & II & I & II \\
\hline ensilage de maïs & & & kg MS & \\
\hline concentré Témoin $(\mathrm{kg})$ & 7,0 & 5,9 & - & - \\
\hline pois protéagineux $(\mathrm{kg})$ & - & - & 6,0 & 5,0 \\
\hline urée $(\mathrm{g})$ & & & & \\
\hline aliment minéral 7-21 (g) & & & & \\
\hline bicarbonate de sodium (g) & & & & \\
\hline phosphate bicalcique (g) & & & & \\
\hline carbonate de calcium (g) & & & & \\
\hline
\end{tabular}

(1) Apport de $1 \mathrm{~kg}$ de foin sur la ration le soir. Complémentation vitaminique réalisée en mélange à la ration complète 2 fois par semaine ( $175000 \mathrm{UI}$ de vit. A, 17500 de vit. D3, $70 \mathrm{mg}$ de vit. E),

tourteau de soja ingérées ont été en moyenne de $0,75 \mathrm{~kg}$ MS. Les teneurs en cellulose brute des 2 rations ont été d'environ 18,5 et $17,2 \%$ et celles d'amidon de 24 et $33 \%$ respectivement.

Les apports d'énergie ont été sensiblement identiques pour les 2 traitements $(15,6$ UFL en moyenne). Les quantités d'azote fermentescible (PDIN) n'ont jamais été limitantes et celles de PDIE ont été inférieures d'environ $115 \mathrm{~g}$ avec le pois protéagineux. Le taux azoté de cette ration (MAT \% MS) a été plus élevé de 1,3 point par rapport au témoin.

\section{* Performances zootechniques et bilans nutritifs}

Les productions n'ont pas été significativement différentes selon les traitements et ont été en moyenne de $23,5 \mathrm{~kg}$ de lait, $948 \mathrm{~g}$ de matières grasses et $692 \mathrm{~g}$ de protéines (tableau 2). L'évolution de la production laitière a été bonne puisqu'elle n'a diminué en moyenne que de $1,5 \%$ par semaine. De même, la composition du lait a été identique mais avec des taux butyreux relativement élevés (près de $41 \mathrm{~g} / \mathrm{kg}$ en moyenne) par rapport au taux protéique $(30 \mathrm{~g} / \mathrm{kg})$. Pour le taux butyreux, ceci peut être dû à l'effet favorable de l'ensilage de maïs sur les synthèses de matières grasses (Hoden et al 1985 ; Hoden et Coulon 1991). Le niveau des apports énergétiques relativement strict par rapport aux besoins des animaux encore en phase de croissance peut expliquer le faible taux protéique (Coulon et Rémond 1991).

Une analyse plus détaillée des résultats selon le niveau de production a été réalisée à partir des $2 \mathrm{X} 6$ vaches les plus fortes productrices de chacun des lots $(31,3 \pm 4,9 \mathrm{~kg}$ de lait par jour en pré-expérience). Les productions de lait $4 \%(+0,9 \mathrm{~kg}$ $\mathrm{P}<0,07)$, de matières grasses $(+55 \mathrm{~g}-\mathrm{P}<0,02)$ et le taux butyreux $(+1,7 \mathrm{~g} / \mathrm{kg}-\mathrm{P}<0,02)$ ont été plus élevées avec la complémentation témoin. Les productions de protéines ainsi que le taux pro- 
Tableau 2. Utilisation du pois protéagineux par les vaches laitières Performances zootechniques ( $2 \times 7$ semaines -10 et 11 vaches)

\begin{tabular}{|c|c|c|c|}
\hline Traitements & Témoin & $\begin{array}{c}\text { Pois } \\
\text { protéagineux }\end{array}$ & ETR \\
\hline \multicolumn{4}{|l|}{ Quantités ingérées ( $k g M S$ ) } \\
\hline ensilage de maïs & 11,2 & 11,6 & \\
\hline foin & 0,8 & 0,8 & \\
\hline concentré Témoin & 5,4 & - & \\
\hline pois protéagineux & - & 4,6 & \\
\hline urée + minéraux & 0,38 & 0,58 & \\
\hline total (\% poids vif) & 3,08 & 3,08 & \\
\hline Apports UFL corrigés ${ }^{(1)}$ & 15,4 & 15,7 & \\
\hline Apports PDI (g) & 1475 & 1360 & \\
\hline MAT \% MS & 13,8 & 15,1 & \\
\hline Lait (kg) & 23,6 & 23,3 & 1,14 \\
\hline Lait $4 \%(\mathrm{~kg})$ & 23,9 & 23,3 & 1,18 \\
\hline Matières grasses (g) & 962 & 934 & 56 \\
\hline Matières protéiques (g) & 694 & 689 & 28 \\
\hline Taux butyreux (\%o) & 41,1 & 40,8 & 1,89 \\
\hline Taux protéique (\%o) & 29,7 & 30,0 & 0,68 \\
\hline Poids vif (kg) & 578 & 570 & \\
\hline \multicolumn{4}{|l|}{ Bilan (apports - besoins) } \\
\hline UFL & $+0,1$ & $+0,7$ & \\
\hline PDI (g) & -55 & -130 & \\
\hline
\end{tabular}

(1) Pour tenir compte des interactions digestives, selon INRA 1988.

Pour les vaches produisant moins de $30 \mathrm{~kg}$ de lait, l'utilisation du pois protéagineux comme concentré unique conduit aux mêmes résultats de production et de composition du lait qu'une complémentation classique. téique n'ont en revanche pas été influencés par la nature de l'aliment concentré. Ceci tendrait bien à montrer une efficacité plus faible de la complémentation à partir du pois protéagineux dans le cas des vaches plus fortes productrices.

Compte tenu du schéma expérimental en inversion avec des durées relativement courtes de chacune des périodes ( 7 semaines) et du nombre de pesées trop réduit, il n'est guère possible d'estimer correctement les variations réelles de poids vif des animaux selon les traitements. Globalement, tous traitements confondus, l'ensemble des animaux n'auraient augmenté leur poids vif que de $10 \mathrm{~kg}$ en moyenne durant la totalité de l'essai (14 semaines) soit un gain apparent d'environ $100 \mathrm{~g} / \mathrm{j}$. Ceci est trop faible pour une majorité de vaches primipares ayant encore des besoins de croissance importants et est à relier à la limitation des quantités ingérées pour des raisons expérimentales.

Sans tenir compte des variations de poids vif, le bilan énergétique a été en moyenne légèrement excédentaire (+ 0,4 UFL), en particulier avec le pois protéagineux $(+0,7$ UFL). A l'opposé, les apports de PDI ont été plus limitants $(-90 \mathrm{~g}$ en moyenne), surtout avec le traitement expérimental $(-130 \mathrm{~g})$. Ceci correspondait bien à l'objectif de l'essai de façon à mieux juger de la valeur azotée pratique du pois protéagineux.

\section{Conclusion Propositions pratiques}

En complément des essais réalisés par ailleurs, cette expérimentation d'utilisation du pois protéagineux comme aliment concentré unique d'une ration complète à base d'ensilage de maïs (+ urée et minéraux) permet de préciser les points suivants :

- Les niveaux d'ingestion correspondent à ceux habituellement observés compte tenu de la limitation des quantités offertes d'ensilage de maïs. En accord avec les autres résultats expérimentaux, le 
pois protéagineux broyé grossièrement peut constituer $30 \%$ de la MS d'une ration d'ensilage de maïs sans entraîner de phénomènes particuliers d'inappétence ou de troubles sanitaires.

- Dans nos conditions expérimentales, par rapport à une complémentation classique témoin réalisée à des niveaux d'apports nutritifs (UFL, PDI) proches de la satisfaction des besoins, un apport iso-énergétique de pois protéagineux (5 à $6 \mathrm{~kg}$ brut) a permis d'obtenir les mêmes performances zootechniques moyennes. Les vaches étaient des primipares produisant près de $30 \mathrm{~kg}$ de lait en début d'essai à environ 6 semaines de lactation. A partir d'une analyse plus détaillée des résultats, ce niveau de production apparaît comme étant la limite supérieure de la bonne valorisation du pois protéagineux en tant que seul aliment complémentaire. Ceci est en accord avec d'autres observations réalisées en particulier dans le cas de l'emploi du lupin blanc doux (Emile et al 1991). De façon générale, les graines protéagineuses ne pourront suffire à elles seules à couvrir les besoins des vaches les plus fortes productrices qui nécessiteront une utilisation simultanée de sources de protéines de qualité (Rulquin 1992). En pratique, avec des rations d'excellente valeur alimentaire permettant de satisfaire les besoins énergétiques, l'utilisation d'une autre source protéique serait nécessaire à partir d'une production de 25 à $30 \mathrm{~kg}$ de lait pour des vaches en milieu de lactation. Ceci laisse donc la possibilité de valoriser des quantités non négligeables de protéines d'origine métropolitaine.

- A l'avenir, d'autres essais tant analytiques que zootechniques devraient être entrepris. Il serait nécessaire de préciser les valeurs PDI des graines protéagineuses à partir de mesures de flux réalisées sur des animaux opérés et de compléter l'approche zootechnique. Des expérimentations plus complètes réalisées à différents niveaux d'apports nutritifs avec des vaches plus fortes productrices à différents stades de lactation permettraient également de mieux connaître les lois de réponse et de proposer des plans de complémentation adaptés.

\section{Références bibliographiques}

CHENAIS F., ESPINASSE R., 1989. Utilisation du pois protéagineux pour la complémentation des vaches laitières. ITEB, EDE 22, UNIP. CR essai n ${ }^{\circ} 89091$.

COULON J.B., REMOND B., 1991. Réponses de la production et de la composition du lait de vache aux variations d'apports nutritifs. INRA, Prod. Anim., 4 (1), 49-56. DUCHATEAU-LAGRANGE C., DEDENON. DENOYELLE N., 1989. Utilisation du pois protéagineux comme source d'azote dans l'alimentation de la vache laitière. UNIP-ITEB-RNED Bovin. CR essai n ${ }^{\circ} 89021$.

EMILE J.C., HUYGHE C., HUGUET L., 1991. Utilisation du lupin blanc doux pour l'alimentation des ruminants : résultats et perspectives. Ann. Zootech., 40, $31-44$.

FAVERDIN P., HODEN A., COULON J.B., 1987. Recommandations alimentaires pour les vaches laitières. Bull. Tech. CRZV Theix, INRA, 70, 133-152

HODEN A., 1982. Valeur nutritive des légumineuses à graines pour les ruminants et utilisation par les vaches laitières. Bull. Tech. CRZV Theix, INRA, 49, 27-32

HODEN A., COULON J.B., DULPHY J.P., 1985. Influence de l'alimentation sur la composition du lait. 3 . Effets des régimes alimentaires sur les taux butyreux et protéique. Bull. Tech. CRZV Theix, INRA, 62, 69-79.

HODEN A., COULON J.B., FAVERDIN P., 1988. Alimentation des vaches laitières, chapitre 8 ; in "Alimentation des Bovins, Ovins et Caprins. Ed. R. Jarrige : INRA Publications, Route de St-Cyr, 78000 Versailles.
HODEN A., COULON J.B., 1991. Maîtrise de la composition du lait : influence des facteurs nutritionnels sur la quantité et les taux de matières grasses et protéiques. INRA Prod. Anim., 4 (5), 361-367.

HUGUET L., HODEN A., MALTERRE L., GEAY Y., MICOL D., BERTIN G., MOURGUET A., 1983. Utilisation des graines de lupin doux par les vaches laitières et les taurillons. Bull. Tech. CRZV Theix, INRA, $54,61-72$.

INRA, 1988. Alimentation des Bovins, Ovins et Caprins. Ed. R. Jarrige : INRA Publications, Route de St-Cyr, 78000 Versailles

RIVIERE F., SOULARD J., 1990. La complémentation des rations à base d'ensilage de maïs pour vaches laitières. CR ITCF-UNIP. La Jaillière n ${ }^{\circ} 8806$

RULQUIN H., 1992. Intérêts et limites d'un apport de méthionine et de lysine dans l'alimentation des vaches laitières. INRA, Prod. Anim., 5 (1) 29 - 36

SAS Institute, Inc., 1987. SAS/STAT Guide for Personnal Computer Version 6 Edition, Cary N.C.

TISSERAND J.L., 1977. Fieldbeans and field peas in ruminant crops. Commission of the European Communities. EUR 5686 EN. p. 273-281.

WEISS P., LEBOUCHER J., 1989. Utilisation de l'ensilage de maìs par des vaches laitières de race normande. Intérêt du pois protéagineux. Pont-Hébert 89-01. 


\section{Summary}

\section{Pulses as unique concentrate for dairy cows}

Two "complete" balanced diets, one based on maize silage, providing the energetic content of a classic control concentrate feed (1 UFL/kg dry matter and 115 PDIE/UFL) and an experimental concentrate based on pulses, were given in limited quantities and compared. Nutritive values (UFLPDI) were predetermined in order to satisfy (without excess) the requirements of the animals. According to an experimental scheme consisting of two 7 week periods, a total of 21 lactating cows (80\% first lactation) divided into two groups received one or the other of these diets. For an average content of $5.4 \mathrm{~kg}$ dry matter of control concentrate or of $4.6 \mathrm{~kg}$ dry matter of pulses, the total dry matter intake were identical (3.08 kg dry matter per $100 \mathrm{~kg}$ live weight). The energetic contents were comparable (15.6 UFL/day) and those of PDI were rather less for the pulse diet. The energy balance was slightly in surplus, especially for the experimental treatment $(+0.7$ UFL). Conversely the PDI balances were deficient, in particular for the pulse $\operatorname{diet}(-130 \mathrm{~g}$ as compared to $-55 \mathrm{~g}$ for the control).

For both treatments, milk production, fat output and protein output were comparable and on average : $23.5 \mathrm{~kg}, 948 \mathrm{~g}$ and $692 \mathrm{~g} /$ day respectively. Milk composition was also identical but protein contents were low with respect to the fat content ( 30 as compared to $41 \mathrm{~g} / \mathrm{kg}$ respectively). However, the most productive cows had a decrease in milk $4 \%$ production $(\mathrm{P}<0.07$, fat output $(\mathrm{P}<0.02)$ and fat content $(P<0.02)$ when they received the pulse diet.

In conclusion, it is possible to valorize large quantities of pulses up to production levels of 25 to $30 \mathrm{~kg}$ of milk per day. To achieve an increase in production, it is necessary to supplement the diet with quality protein.

HODEN A., DELABY L., MARQUIS B., 1992. Pois protéagineux comme concentré unique pour vaches laitières. INRA Prod. Anim., 5 (1) 37 - 42 . 